\title{
回转副精度特性的弹性圥余空间机构模型"
}

\author{
王德伦 ${ }^{1}$ 王 智 ${ }^{1}$ 董惠敏 $^{1}$ 于树栋 $^{1,2}$ \\ (1. 大连理工大学机械工程学院 大连 116024;
}

2. 瑞尔森大学机械与工业工程系 多伦多 M5B $2 \mathrm{~K} 3$ 加拿大)

\begin{abstract}
摘要: 提出回转副精度特性的弹性冗余空间机构模型, 将实际回转副中的弹性约束等效映射为多个从动件弹性几余支承, 实 际回转副零件的几何误差特性等效映射为刚性凸轮轮廓曲面, 形成以多组从动件弹性悬浮刚性凸轮几何轮廓的弹性几余空间 机构, 从而较真实地反映实际回转副结构及其零件的几何、运动、材料物理性质与工况载荷特性等因素的耦合关系。建立弹 性冗余空间凸轮机构的几何位移基本方程、静力平衡方程、物性方程, 将回转副精度及其随位置和载荷变化的特性转化为弹 性冗余空间机构的位移分析与静力分析求解问题, 并给出典型回转副精度特性计算分析与试验测试对比示例, 为机器精度分 析与设计提供新的理论基础, 也为机构学理论开辟新的应用领域。
\end{abstract}

关键词: 机构 精度 回转副 弹性约束 几余约束

中图分类号: TG156

\section{A Novel Precision-characterization Model of a Revolute Joint Based on the Concept of Spatial Mechanisms with Redundant Elastic Constraints}

\author{
WANG Delun ${ }^{1} \quad$ WANG Zhi $^{1}$ DONG Huimin ${ }^{1} \quad$ YU Shudong ${ }^{1,2}$
}

(1. School of Mechanical Engineering, Dalian University of Technology, Dalian 116024;

2. Department of Mechanical and Industrial Engineering, Ryerson University, Toronto M5B 2K3, Canada)

\begin{abstract}
A novel approach, based on the concept of spatial mechanism with redundant elastic constraints is presented to set up a comprehensive precision-characterization model of an actual revolute joint. When considering component flexibility and manufacturing errors, the shaft in an actual revolute joint can move in a much more complex manner in comparison with an ideal revolute joint with only one rotational degree of freedom. In the proposed model, the rigid cams with special profiles are used to represent the geometrical error, springs are used to describe the deformations of joint components and surfaces in actual machine parts. The spatial cams, suspended by multiple straight-followers with springs, called an RE mechanism, is proposed to simulate the kinematics of the shaft in the revolute joint. The basic model equations, such as kinematic equations, equations of equilibrium, and equations of physical properties are concisely derived. With the proposed model, the precision of an actual revolute joint and its load-dependent properties can be seen as the solutions of the basic equations of a corresponding RE mechanism. An example is presented to illustrate the solution process of the precision model of a revolute joint, and numerical results were compared with the experimental results obtained from the corresponding test setup. The proposed model provides for the first time a theoretical basis for joint precision analysis and synthesis. This opens up a new application of the theory of mechanism in design of machine components.
\end{abstract} Key words: Mechanism Error Revolute joint Elastic constraint Redundant constraint

\section{0 前言}

机构抽象反映了机器中构件间的运动与力的 传递及变换关系, 是机器运动与尺度设计的基本内

*国家自然科学基金资助项目(51275067)。20121129 收到初稿, 20130621 收到修改稿
容; 机构中的构件是刚体，运动副是理想形状；机 构中的理想回转副, 有 5 个自由度被刚性约束, 只 有 1 个回转自由度。然而, 实际机器中的运动副是 由两个或多个实际几何曲面及介质支承接触形成, 不仅每个曲面有制造误差，而且还有弹性，会产生 变形; 同时, 运动副元素所属的构件是由若干弹性 零件联接而成, 在载荷作用下会产生变形, 也影响 
运动副元素的几何曲面形状和接触位置; 所以, 机 构中的构件和运动副都存在制造误差和弹性变形, 使得实际机器与理想机器中的零部件位置及运动产 生差异。这种差异及其在载荷作用下的变化特性形 成了机器的精度及其特性。而含有误差的弹性零件 曲面及介质构成的实际回转副, 与理想运动副约束 的偏差及其变化性质, 构成实际回转副的精度与 特性。

机器精度及其特性是机器性能的重要指标, 如 机床和精密仪器仪表等, 精度丧失意味着机器失效。 然而, 机器精度主要包括构件精度(零件及其静态联 接面)与运动副精度; 那么, 构件及运动副精度与其 零件及联接有何关系? 如何计算与评价? 在运动状 态与工况载荷作用下的特性是什么? 这些问题目前 尚待研究; 现有机器精度研究文献也有采用机构模 型, 有的将零件与动联接面的误差特征表述为机构 的刚性杆长误差与运动副间隙 ${ }^{[1-7]}$, 有的直接用机构 表示误差 ${ }^{[8]}$, 有的赋予机构构件弹性参数 ${ }^{[9]}$, 也有 利用机构学原理进行零件误差坐标变换或矩阵变 换 ${ }^{[10-13]}$; 这些研究多是把机器精度简化为机构刚性 构件精度或几何精度, 部分考虑了构件弹性变形, 但缺少运动副结构、零件几何误差、材料物理性能 与载荷之间的有机联系, 难以体现不同运动状况和 载荷作用下机器的精度变化特性。实际构件及运动 副是通过多个具有误差的几何表面间弹性接触与变 形协调、或相对运动来实现运动与力的传递, 其精 度及特性是结构、几何、运动、弹性与工况载荷之 间耦合关系的综合体现。

回转副是机器中常用的运动副, 如机床主轴, 一般由轴、轴承等运动零件与轴承座等支承固定零 件组成。本文总结实际回转副的常见结构类型, 分 析其弹性支承约束与几何精度映射, 构建回转副精 度特性的弹性几余空间机构模型, 讨论该模型的基 本方程及其性质, 把实际回转副的精度特性问题转 化为弹性冗余空间机构的位移与静力求解问题, 从 而形成回转副精度特性模型, 对于涉及温度、动态、 时变等因素时该模型的修正和其他运动副的精度特 性模型, 作者将另文探讨。

\section{1 回转副典型轴承结构与弹性约束}

实际回转副是由具体轴承结构及介质实现对 旋转轴运动的支承与约束, 主要有滚动轴承和滑动 轴承及其组合; 这些轴承依据承载方向不同呈现向 心轴承、推力轴承、向心推力 (或推力向心)轴承等 结构类型; 以滚动轴承及其组合为例, 具体典型结
构与轴承约束分别讨论如下。

\section{1 回转副的滚动轴承结构}

为了实现回转副 5 个自由度约束, 一般都有两 个以上轴承组合使用, 其典型组合安装方式有双支 点单向固定与一端固定另一端游动两种形式, 以深 沟球轴承为例的典型结构, 如图 1 和图 2 所示。

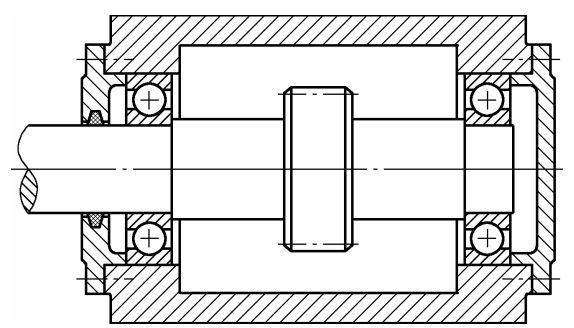

图 1 回转副对称双支点单向固定滚动轴承结构

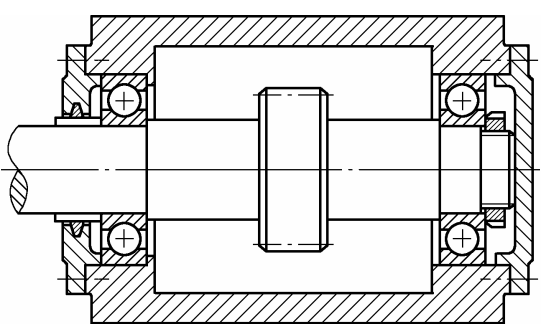

图 2 回转副一端固定一端游动滚动轴承结构

将深沟球轴承替换为其他型式的轴承, 如推力 球(滚子)轴承、圆锥滚子轴承、圆柱滚子轴承、调 心球(滚子)轴承等, 并适当修改定位结构, 可得到 类似的回转副滚动轴承结构。如以一对角接触球轴 承代替图 2 中左端轴向固定的深沟球轴承, 而以圆 柱滚子轴承代替右端的深沟球轴承, 形成承受更大 轴向和径向载荷的回转副结构, 如图 3 所示的机床 主轴类似结构。

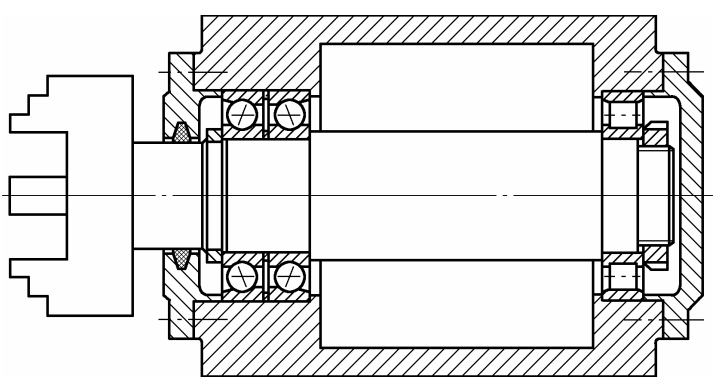

图 3 回转副角接触球轴承与圆柱滚子轴承结构

显然, 不同类型结构的滚动轴承组合具有不同 的约束性质与刚度, 从而得到不同的回转副精度及 其特性。

\section{2 回转副的滚动轴承约束与刚度}

对于回转副的滚动轴承结构, 由于滚动轴承可 以向心与推力轴承结构集成在一起, 使得滚动轴承 的功能集成化, 其结构多样化且约束复杂化; 在此 仅论述四类滚动轴承。 
向心轴承: 有深沟球轴承和圆柱滚子轴承, 如 图 4a 所示, 前者主要承受径向载荷, 也可承受一定 的双向轴向载荷, 即约束 2 个径向自由度(用实线弹 簧表示)和 1 个轴向自由度(用虚线弹簧表示), 其中 轴向约束与轴承轴向定位结构相关。而后者仅承受 径向载荷, 即约束 2 个自由度径向位移(用实线弹簧 表示), 其他方向需要有辅助支承限制, 即需要组合 使用; 在此约定: 单方向弹性约束采用单个弹簧 $k_{j}^{V}$ 表示, 上标 $V(V=X, Y, Z)$ 表示弹簧约束的坐标轴方 向, 下标 $j$ 表示沿坐标轴 $V$ 的正向或反向, $j=1$ 表示 沿坐标正向, $j=2$ 表示反向; 后文均采用相同约定。

推力轴承: 无论滚动体是球还是滚子, 见图 $4 \mathrm{~b}$, 仅承受单向轴向载荷, 也就是约束一个单向自由度 (用实线弹簧表示), 需要与其他轴承组合使用。

向心推力或推力向心轴承: 有角接触球轴承和 圆雉滚子轴承, 见图 $4 \mathrm{c}$, 都可同时承受径向载荷和 单向轴向载荷, 即约束 2 个径向自由度(用实线弹簧 表示)和 1 个单向轴向自由度(用实线弹簧表示), 一 般需要成对组合使用。

调心轴承: 有调心球轴承和推力调心滚子轴 承, 允许轴线偏转, 见图 $4 \mathrm{~d}$; 前者主要承受径向载 荷, 也可承受一定的双向轴向载荷, 即约束径向和 轴向位移(3 个自由度); 后者则以轴向载荷为主, 也 可承受径向载荷, 即约束 2 个径向自由度和双向轴 向自由度(用实线弹簧表示)。

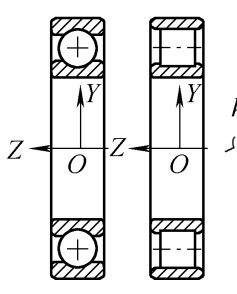

(a)

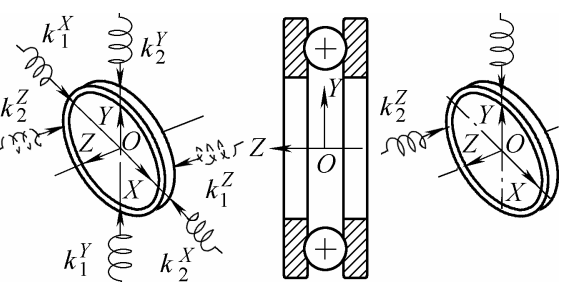

(b)
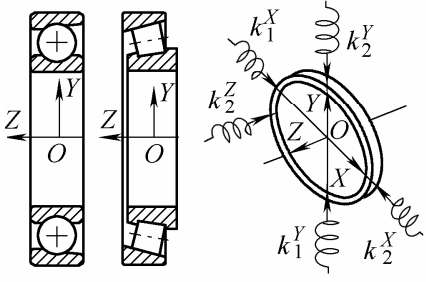

(c)
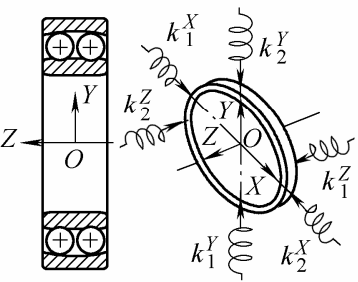

(d)
图 4 四类典型滚动轴承结构与约束

为简化表示, 图 4 中的约束模型仅给出了实现 上述轴承约束所需的最少约束弹簧数; 实际滚动轴 承的支承约束与滚道、滚动体之间的接触状态相关, 轴向约束与径向约束之间也存在关联性, 可根据分 析需要适当增加约束弹簧的数量或改变约束弹簧的 位置, 如图 $4 \mathrm{c}$ 中向心推力轴承可采用 4 个与径向约
束弹簧一一对应的轴向约束弹簧。

回转副中旋转轴的弹性位移是轴承座、轴承、 轴等零部件及其配合面弹性变形的累积, 若以旋转 轴上轴承支承段中心截面的形心 $O$ 为参考, 上述轴 承约束等效弹簧刚度 $\left(k_{j}^{V}\right)$ 可定义为截面形心 $O$ 沿轴 线 $V(V=X, Y, Z)$ 方向上的载荷与弹性位移比值, 不同 方向上等效弹簧刚度不同。可对轴承进行适当简化, 建立回转副的整体有限元模型 ${ }^{[14]}$ 计算等效约束弹 簧刚度。由于滚动轴承一般为标准件, 为计算方便 也可分为轴承座与轴承两部分近似简化计算, 轴承 刚度可按滚动轴承技术手册提供的资料计算, 如单 列向心球轴承的径向刚度 ${ }^{[15]}$

$$
k_{B}^{r}=32375 Z D_{w}^{1 / 2} \delta_{r}^{1 / 2} \cos ^{5 / 2} \alpha
$$

式中, $Z$ 为滚动体个数, $D_{w}$ 为滚动体直径, $\delta_{r}$ 为径 向弹性变形, $\alpha$ 为接触角。

\section{3 回转副滚动轴承结构的弹性约束等效模型}

由上述可知, 回转副旋转轴的位置精度及其特 性与轴承支承结构及约束性质相关, 也就是与轴承 类型与轴承安装方式相关, 在此以图 1 3 的典型回 转副结构作为示例阐述。

如图 1 所示的回转副结构, 将旋转轴由若干支 承截面弹性悬浮起来, 形成回转副轴承结构的物理 性质等效——弹性约束等效模型，左右两端轴承分 别等效为两个径向弹性约束和一个单向轴向弹性约 束，并以弹簧表示，依据弹性约束性质，这是最少 约束数目, 见图 5。虽然在实际运转过程中, 轴承 弹性约束可以在 $360^{\circ}$ 任意方向, 在此模型中分解为 $X, Y, Z$ 三个方向, 旋转轴的空间位置和静态平衡状 态由各弹簧刚度及各支承截面的几何形状确定。

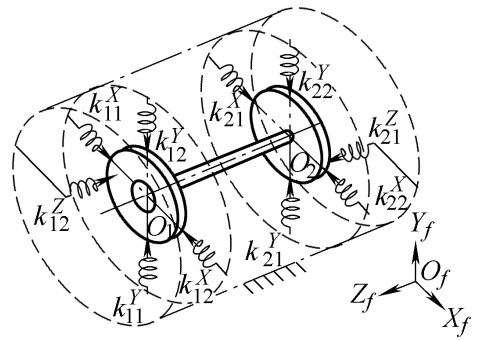

图 5 回转副双支点单向固定结构弹性约束模型

而图 2 所示的回转副结构, 其右端轴承有 2 个 径向弹性约束自由度(没有轴向弹性约束), 左端有 2 个径向弹性约束自由度和 1 个双向轴向弹性约束, 将弹性约束以弹簧表示, 属于一端固定一端游动结 构的弹性约束模型，如图 6 所示。

按照第 1.2 节的轴承约束分析, 回转副的不同 轴承组合结构, 可以按照轴承类型和安装结构的约 束性质，以弹簧表示弹性约束，构建回转副的弹性 


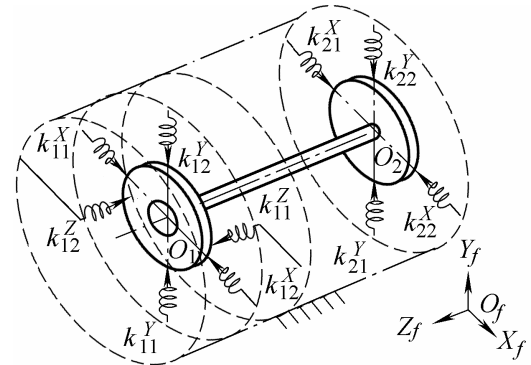

图 6 回转副一端固定一端游动结构弹性约束模型

约束模型。如图 3 所示的回转副轴承结构, 由向心 圆柱滚子轴承与双排角接触球轴承(向心推力轴承) 组合形成, 即右端轴承有两个径向弹性约束(没有轴 向弹性约束), 左端有双排径向弹性约束和双向轴向 弹性约束, 同属于一端固定一端游动结构的弹性约 束模型, 如图 7 所示, 对于多排的滚动轴承约束, 只需要增加对应的轴承支承约束截面即可, 并考虑 多组轴承组合对轴承约束刚度的影响 ${ }^{[16]}$ 。

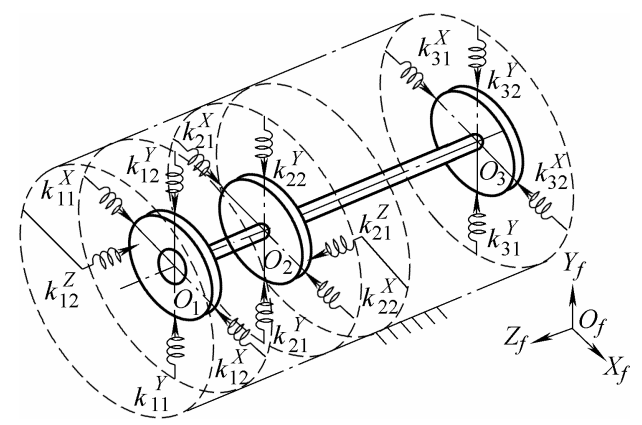

图 7 回转副一端双固定一端游动结构弹性约束模型

\section{2 回转副精度的弹性冗余机构模型}

对于回转副滚动轴承结构而言, 其旋转轴在运 动或承受载荷时, 若干支承在不同方位有几何误差 和弹性约束, 约束的类型和程度取决于轴承的类型 和结构, 由于弹性约束及其变化规律与具体结构、 几何参数、材料、工况载荷等条件相关, 从而决定 了回转副的精度及其特性, 几何误差需要等效映射 以便简化几何计算模型, 但几何误差和弹性变形在 安装和工作载荷作用下往往会相互转化, 因此, 需 要建立涵盖几何映射和弹性约束的回转副精度弹性 几余机构模型。

\section{1 回转副轴承结构的运动与几何等效}

回转副轴承结构的旋转轴由若干支承截面弹 性悬浮起来后, 当轴承运动联接面存在几何误差时, 外圈相对固定, 旋转轴(含内圈)的轴线方向与位置 随内圈位置变化而改变, 其与理想位置及方向的差 别综合反映了轴承运动联接面几何误差对旋转轴位 置与运动精度的影响。在此构造一组等效刚性凸轮
轮廓, 该凸轮与旋转轴固接, 使凸轮与旋转轴该截 面形心具有相同的运动规律一一几何与运动等效; 由于旋转轴的位置与运动由轴承元件几何形状(含 误差)和受力平衡确定, 运动等效需要满足几何等效 条件和力平衡条件, 即旋转轴的空间位置、运动和 静态平衡状态由等效凸轮轮廓几何形状与各弹簧刚 度确定, 若等效凸轮轮廓在任意位置均满足等效运 动的几何条件和等效支承约束反力与外载荷的平衡 条件, 则该凸轮可以等效描述轴承旋转轴的空间几 何位置与运动。

滚动轴承支承结构几何误差及其特性主要包 括零件表面几何误差及其特征、两零件表面固定(静) 联接几何误差特征传递、两个或多个零件表面运动 (动)联接几何误差特征传递三方面, 即等效刚性凸 轮轮廓体现零件曲面几何误差特征及其静动联接面 传递, 如图 8 所示。

（1）零件表面几何误差及其特征, 如内圈内孔 表面、滚道表面的几何误差特征等; 回转副零件表 面实际几何特征可描述为理想曲面附加几何误差特 征的形式, 以曲面方程描述

$$
S: r=S(\theta, z)=S_{0}(\theta, z)+\mu(\theta, z)
$$

式中, $r 、 \theta 、 z$ 为坐标变量, $r=S_{0}(\theta, z)$ 为理想 曲面, $r=\mu(\theta, z)$ 为几何误差特征。可测量零件表 面若干截面轮廓, 并以曲线拟合得到各截面轮廓曲 线, 如采用傅里叶级数拟合截面 $i$ 的曲线方程

$$
r=r_{i 0}+a_{i 0}+\sum_{n}\left[a_{i n} \cos \left(n w_{i} \theta\right)+b_{i n} \sin \left(n w_{i} \theta\right)\right]
$$

式中, $r 、 \theta$ 为坐标变量, $r_{i 0}$ 为截面 $i$ 理想形状对 应的基值， $a_{i 0} \sim a_{i n} 、 b_{i 1} \sim b_{i n} 、 w_{i}$ 为拟合参数，对应 截面几何误差特征; 以所有被测截面的轮廓曲线数 据为基础, 通过曲面拟合得到零件表面轮廓, 在此 以 $\Delta(S)$ 表示拟合曲面 $S$ 的几何误差特征。

(2) 零件表面 $S_{A}$ 和零件表面 $S_{B}$ 通过固定(静)联 接方式 $\xi$ 将其几何误差特征进行传递映射到另一工 作表面 $S_{g}$, 有

$$
\xi:\left(\Delta\left(S_{A}\right), \Delta\left(S_{B}\right)\right) \rightarrow \Delta\left(S_{g}\right)
$$

如图 $8 \mathrm{~b}$ 所示, 轴、内圈内孔几何误差特征通 过内圈与轴的过盈配合传递与映射到内圈滚道。

(3) 两个或多个零件表面 $S_{A}, S_{B}, S_{C}, \cdots$ 通过运 动(动)联接方式 $\eta$ 将其几何误差特征进行映射与传 递转换为运动零件的轨迹变化 $\delta$, 有

$$
\eta:\left(\Delta\left(S_{A}\right), \Delta\left(S_{B}\right), \Delta\left(S_{C}\right)\right) \rightarrow \delta
$$




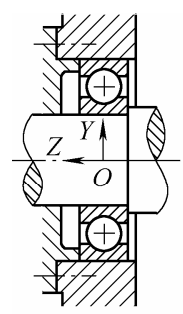

(a)

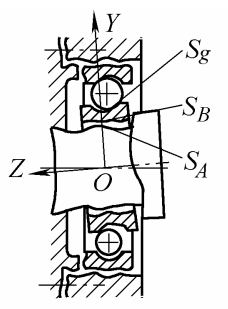

(b)

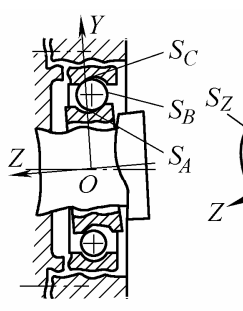

(c)

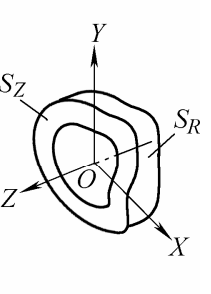

(d)
图 8 回转副滚动映射曲面示意图

如图 $8 \mathrm{c}$ 所示内圈滚道、外圈滚道和滚动体的几 何误差特征通过相对运动传递映射给内圈位置与 运动。

显然, 对于回转副滚动轴承结构, 既有旋转轴、 轴承元件、轴承座等零件表面的几何误差, 又有轴 与内圈、外圈与轴承座的静联接 $\xi$ 以及内圈、外圈 和滚动体的动联接 $\eta$ 的误差特征传递与映射; 以内 圈滚道面为凸轮轮廓基础, 以相关曲面误差特征函 数经过静、动联接面传递与映射为依据, 构建等效 凸轮的轮廓, 其径向与轴向轮廓方程可分别表述为

$$
\begin{aligned}
& S_{R}: r=S_{R}(\theta, z)=r_{0}(\theta, z)+\delta_{R}(\theta, z)+\sigma_{R}(\theta, z) \\
& S_{Z}: z=S_{Z}(r, \theta)=z_{0}(r, \theta)+\delta_{Z}(r, \theta)+\sigma_{Z}(r, \theta)
\end{aligned}
$$

式中, $r 、 \theta 、 z$ 为坐标变量, $r_{0}(\theta, z) 、 z_{0}(r, \theta)$ 对 应内圈滚道曲面理想轮廓, $\delta_{R}(\theta, z) 、 \delta_{Z}(r, \theta)$ 为曲 面误差特征经过静、动联接面传递与映射后在凸轮 坐标系中的像, $\sigma_{R}(\theta, z) 、 \sigma_{Z}(r, \theta)$ 为凸轮轮廓的载 荷特征系数。

轴承等效支承约束模型中, 每个单向支承约束 与其对应凸轮轮廓构成一个单向弹性约束, 一组共 线支承约束与一对共轭凸轮轮廓构成一个自由度约 束，即 10 个支承约束对应 10 个凸轮轮廓，构成 5 个自由度约束, 满足任意位置上的约束与力平衡条 件。由于篇幅所限, 回转副滚动轴承结构对应的具 体凸轮方程的形成过程与参数将另文论述。

在凸轮坐标系 $(O X Y Z)_{i}$ 下将凸轮轮廓方程记为

$$
S_{i j}^{V}: g_{i j}^{V}(x, y, z)=0 \quad i=1,2 \quad j=1,2 \quad V=X, Y, Z
$$

式中, $i, j 、 V$ 含义与弹性约束中约定一致。回转副 在不同工况与载荷作用下, 回转轴将产生弹性变形, 即两端各等效凸轮曲面之间有相对弹性位移, 两端 凸轮之间存在六个相对位移参数, 在后文讨论。

一般而言, 滚动轴承为标准件, 可以通过研究 滚动轴承的精度特性, 简化为带有映射函数的凸轮 轮廓几何曲面, 外部(轴与轴承座)曲面的几何特征 可以作为映射函数的输入参数, 这是一项具有重要 意义的研究工作, 还需要深入研究。

\section{2 回转副精度的弹性几余机构模型}

采用上述弹性约束与几何特征映射简化模型,
可以表示瞬时位置回转副的状态, 而在任一位置或 连续运动且不同工况载荷情况下，需要建立回转副 精度与特性的数学模型。因此, 考虑到几何、运动 与静力平衡等方面的连续性, 提出将旋转轴上的等 效映射几何曲面转化为刚性盘状凸轮 $S_{i j}^{V}$, 而对旋转 轴曲面的弹性支承约束等效为带有弹簧预紧恢复力 的刚性滚子直动从动件 $k_{i j}^{V}$ 作用于刚性凸轮, 使得每 个轴承简化为带弹簧复位的多从动件凸轮机构; 为 便于计算, 将从动件导轨分布在机架坐标系中的 $X$ 、 $Y 、 Z$ 轴上(或平行); 对于回转副的多轴承组合结构, 如图 1 所示的双支点单向固定回转副轴承结构, 可 等效为如图 9a 所示的多从动件弹性支承的刚性凸 轮机构; 回转副中由结构弹性引起的位移或自由度 称为弹性自由度, 而这种弹性自由度是冗余的, 在 实际回转副轴承结构中又必然存在, 即弹性几余自 由度，上述机构称为弹性支承多自由度攵余空间凸 轮机构, 简称为弹性元余机构。

弹性攵余机构的结构与性能参数集中体现了 实际回转副轴承结构的几何误差与弹性的影响：等 效凸轮轮廓综合体现了相关零件的几何误差与预紧 受力状态，从动件复位弹簧在机架上位置反映了轴 承座位置误差以及轴承外圈与轴承座之间配合状况 的影响, 从动件复位弹簧反映了轴承结构与接触刚 度，两端凸轮之间弹簧 $C_{S}$ 表示了回转轴的弹性等, 该模型综合反映了实际回转副结构、几何、物理、 载荷等因素之间的耦合关系。

对于如图 2 所示的一端固定另一端游动回转副 轴承结构, 可得到另一种弹性几余空间机构, 如图 $9 \mathrm{~b}$ 所示。对于不同轴承类型与结构参数的回转副, 可得到相应弹性约束形式从动件的个数、方向、位 置以及凸轮几何轮廓、形状与相对位置等，从而建 立回转副精度特性的弹性冗余机构模型。

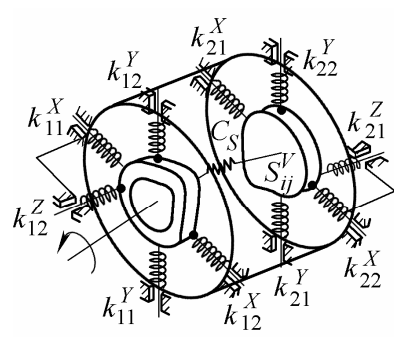

(a)

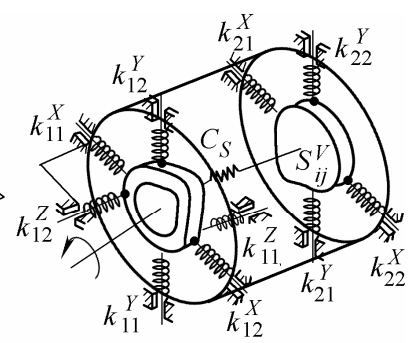

(b)
图 9 回转副精度的弹性几余机构模型

\section{3 弹性圥余机构的基本方程}

在弹性冗余机构中，凸轮及其旋转轴转动任何 
角度, 其空间位置(轴线)由径向与端面凸轮几何轮 廓及其从动件弹簧(含旋转轴变形)协调确定, 可以 通过弹性冗余机构的位移方程与静力平衡方程解 出。在此分析回转副精度特性的弹性元余机构的自 由度, 建立位移与静力基本方程。

\section{1 弹性冗余机构自由度分析}

如图 9a 所示的双支点单向固定滚动轴承结构 对应的弹性几余空间机构, 简称双支点弹性冗余空 间机构, 共有 12 个构件, 即 1 个回转轴(带有两个 凸轮), 10 个直动从动件和 1 个机架; 而每个从动 件分别与机架构成一个移动副、与凸轮构成一个高 副, 共 10 个 I 级副和 10 个 $\mathrm{V}$ 级副, 那么, 由机构 自由度计算公式 ${ }^{[17]}$ 可知

$$
F=6(n-g-1)+\sum_{i=1}^{g} f_{i}
$$

将构件数 $n=12$ 、运动副数 $g=20$ 以及 10 个 I 级副和 10 个 $\mathrm{V}$ 级副代入上式可以得到

$$
F=6 \times(12-20-1)+1 \times 10+5 \times 10=6
$$

即双支点弹性几余机构有 6 个自由度, 其中凸轮(轴) 回转是 1 个绝对自由度, 其余 5 个是由于材料弹性 变形引起的弹性自由度, 表现为凸轮应该被约束的 3 个方向的线位移和 2 个方向的角位移, 与旋转轴 悬浮起来的支承运动相对应。

双支点弹性冗余机构的 10 个从动件对应 10 个 弹性运动, 对于刚体机构而言, 5 个从动件即可完 全描述了刚性凸轮的 5 个自由度运动, 另外 5 个从 动件是圥余弹性运动, 也是最少冗余弹性运动, 从 另一角度说明了弹性冗余的含义。

对于一端固定一端游动结构的回转副, 弹性几 余空间机构的自由度也一样。

显然, 该机构中每增加一个刚性从动件会引入 6 个自由度, 同时也带来一个 I 级副(与凸轮构成高 副)和一个 $V$ 级副(移动副), 即 6 个约束, 正好抵消, 或者说增减从动件及其移动副与高副, 不会改变回 转副弹性几余机构的自由度数, 即最简化弹性冗余 机构。这为分析含有结构误差与过约束或超静定轴 承结构提供便利。

值得讨论的是, 在传统机构学中, 约束与自由 度是相互对立而又相互依存, 自由度是相对机架而 言的; 同时, 构件的自由度数目又是独立运动参数 的数目, 固定为机架的构件没有独立运动参数 (或自 由度为 0 )。但在实际机器中, 机架上各点因材料受 力弹性变形而被动产生了弹性位移或运动, 使被约 束构件具有了弹性运动自由度, 与刚体机构中原构 件运动规律或机构自由度无关, 这是弹性冗余自由
度与传统机构学刚体构件中自由度含义的区别。

\section{2 弹性几余机构的位移方程}

以图 9a 所示的双支点弹性几余空间机构为例, 分别建立直角坐标系, 如机架 $(O X Y Z)_{f}$ 、输入截面 $(O X Y Z)_{P 2}$ 、输出截面 $(O X Y Z)_{P 1} 、 2$ 个等效凸轮 $(O X Y Z)_{1}$ 与 $(O X Y Z)_{2} 、 10$ 个直动从动件 $(O X Y Z)_{i j}^{V}(i, j=1,2 ; V=X$, $Y, Z)$; 在此约定: 字母上标 $X 、 Y 、 Z$ 表示位移方向, 下标 $i$ 为凸轮(截面)序号, 下标 $j$ 表示在该运动方向 上的从动件序号; 其中 $i=1$ 表示从属左端凸轮曲面, $i=2$ 表示从属右端凸轮曲面, 如图 10 所示。则从动 件坐标原点 $O_{i j}^{V}$ 相对于机架坐标原点 $O_{f}$ 的位移表示 凸轮从动件的(弹性)位移, 即轴承结构的弹性变形; 凸轮坐标原点 $O_{i}$ 相对机架坐标原点 $O_{f}$ 的位移表示凸 轮中心的运动, 即回转副轴心的位移, 而凸轮坐标 原点 $O_{2}$ 相对于 $O_{1}$ 的位移表示旋转轴的弹性变形。

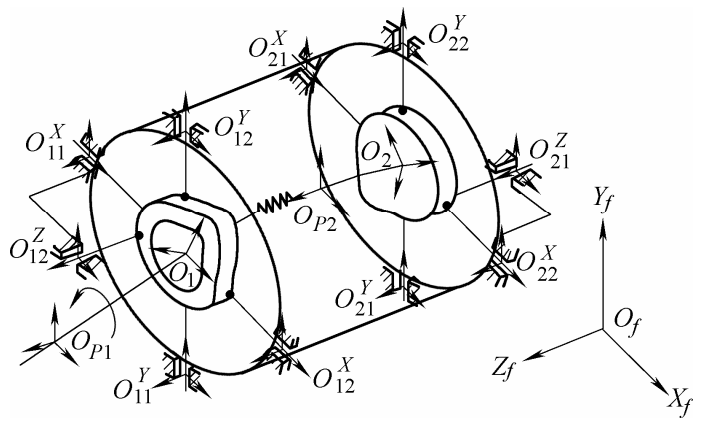

图 10 弹性几余机构的坐标系

将凸轮轮廓方程式(8)置于机架坐标系 $(O X Y Z)_{f}$ 中, 得到在固定坐标系的方程

$g_{i j}^{V f}\left(x, y, z, \boldsymbol{R}_{i}\right)=0 \quad i=1,2 \quad j=1,2 \quad V=X, Y, Z(10)$ 式中, 参数 $\boldsymbol{R}_{i}=\left(x_{i}, y_{i}, z_{i}, \theta_{x i}, \theta_{y i}, \theta_{z i}\right)$ 决定凸轮 $i$ 在坐 标系 $(O X Y Z) f$ 中的位置与姿态。

凸轮轮廓曲面与十个从动件导轨轴线分别相 交, 三向导轨轴线方程在机架坐标系下可分别写为

$$
\begin{gathered}
y-y_{i j}^{X f}=z-z_{i j}^{X f}=0 \quad i=1,2 \quad j=1,2 \\
x-x_{i j}^{Y f}=z-z_{i j}^{Y f}=0 \quad i=1,2 \quad j=1,2 \\
x-x_{i j}^{Z f}=y-y_{i j}^{Z f}=0 \quad i=1 \quad j=2 \text { 或 } i=2 \quad j=1
\end{gathered}
$$

可得到凸轮曲面上十个交点, 即凸轮轮廓与从动件 $k_{i j}^{V}$ 的接触点 $P_{i j}^{V f}$, 在坐标系 $(O X Y Z)_{f}$ 下的坐标记为 $\left(x_{i j}^{V f}, y_{i j}^{V f}, z_{i j}^{V f}\right)$, 接触点位置随凸轮轮廓形状和凸轮 位置而变化。而凸轮轮廓几何曲面在接触点处的法 矢由曲面方程式(10)求得

$$
\boldsymbol{n}_{i j}^{V}=\left(\frac{\partial g_{i}^{f}}{\partial x}, \frac{\partial g_{i}^{f}}{\partial y}, \frac{\partial g_{i}^{f}}{\partial z}\right)=\left(n_{i j}^{V x}, n_{i j}^{V y}, n_{i j}^{V z}\right)
$$

显然, 凸轮轮廓上接触点处的法线矢量 $\boldsymbol{n}_{i j}^{V}$ 与从 
动件移动方向 $\boldsymbol{v}_{i j}^{V}$ (分别沿 $X_{f} 、 Y_{f} 、 Z_{f}$ 轴方向)存在夹 角 $\alpha_{i j}^{V}$, 称为凸轮机构在该点的压力角

$$
\cos \alpha_{i j}^{V}=\frac{\boldsymbol{n}_{i j}^{V} \cdot \boldsymbol{v}_{i j}^{V}}{\left|\boldsymbol{n}_{i j}^{V}\right| \times\left|\boldsymbol{v}_{i j}^{V}\right|}
$$

与实际轴承的接触角、载荷类型与方向等有关, 在 凸轮几何曲面等效时需要考虑。

为便于建立凸轮机构的位移方程, 在此建立位 移矢量。从动件 $k_{i j}^{X}$ 复位弹簧在空载状态下, 从动件 端点到从动件与机架移动副接触点 $O_{i j}^{V}$ 之间的矢量 记为 $\boldsymbol{L}_{i j}^{V} ; \boldsymbol{\delta}_{i j}^{V}$ 表示从动件 $k_{i j}^{X}$ 复位弹簧在工作载荷下 的变形量; $\boldsymbol{r}_{i j}^{V}$ 表示凸轮中心 $O_{i}$ 到接触点 $P_{i j}^{V f}$ 的矢 量, 如图 11 所示; 以 $O_{f i}$ 表示第 $i$ 个凸轮截面上从 动件移动 $X$ 导轨轴线与 $Y$ 导轨轴线的交点, 仅与机 架相关, $\boldsymbol{r}_{i}$ 表示 $O_{f i}$ 到 $O_{i}$ 的矢量。

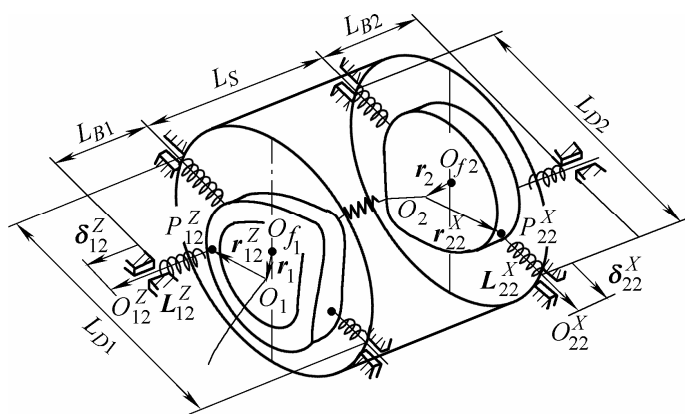

图 11 弹性午余机构的位移示意图

弹性冗余机构中构件与机架为刚体, 从动件复 位弹簧作用于机架使从动件与凸轮保持接触, 机架 两固结参考点之间的距离为 $L_{D i}$, 对应于轴承曲面的 中心截面公称直径, 如滑动轴承的轴瓦曲面或滚动 轴承的外圈滚道; 回转副的两轴承支承面之间的距 离为 $L_{S}$, 对应回转副两端轴承中心截面的距离; 轴 向从动件与机架上作用点到支承截面的距离为 $L_{B i}$, 对应回转副轴向定位端面到轴承中心截面的距离; 上述距离均为机架的固定参数, 与机构运动参数无 关。分别建立弹性几余空间机构在任一支承截面的 $X 、 Y 、 Z$ 方向的位移基本方程

$$
\begin{gathered}
\left|\boldsymbol{r}_{i}+\boldsymbol{r}_{i j}^{X}+\left(\boldsymbol{L}_{i j}^{X}-\boldsymbol{\delta}_{i j}^{X}\right)\right|=L_{D i} / 2 \quad i=1,2 \quad j=1,2 \\
\left|\boldsymbol{r}_{i}+\boldsymbol{r}_{i j}^{Y}+\left(\boldsymbol{L}_{i j}^{Y}-\boldsymbol{\delta}_{i j}^{Y}\right)\right|=L_{D i} / 2 \quad i=1,2 \quad j=1,2 \\
\left|\boldsymbol{r}_{i}+\boldsymbol{r}_{i j}^{Z}+\left(\boldsymbol{L}_{i j}^{Z}-\boldsymbol{\delta}_{i j}^{Z}\right)\right|=L_{B i} \quad i=1 \quad j=2 \text { 或 } i=2 \quad j=1(18
\end{gathered}
$$

回转副轴承结构的等效弹性几余机构有 8 个径向位 移基本方程, 2 个轴向位移基本方程, 而其中待求 变量包括 10 个弹性变形量 $\boldsymbol{\delta}_{i j}^{V}$ 以及 $\boldsymbol{r}_{i}$ 对应的 6 个坐 标变量。共有 10 个基本方程, 16 个待求变量。

\section{3 弹性几余机构静力平衡方程}

实际回转副在工作时的外力, 包括驱动力和工 作阻力, 均可以有多组, 可顺次将力与力矩记为 $\boldsymbol{F}_{P 1} \sim \boldsymbol{F}_{P n} 、 \boldsymbol{M}_{P 1} \sim \boldsymbol{M}_{P n}$, 在机架坐标系 $(O X Y Z)_{f}$ 中描 述为 $\boldsymbol{F}_{P n}=\left(F_{P n}^{X}, F_{P n}^{Y}, F_{P n}^{Z}\right) 、 \boldsymbol{M}_{P n}=\left(M_{P n}^{X}, M_{P n}^{Y}, M_{P n}^{Z}\right)$, 可等效作用在点 $O_{P n}$, 机架坐标系原点 $O_{f}$ 到 $O_{P n}$ 的 矢量记为 $\boldsymbol{r}_{P n}=\left(x_{P n}, y_{P n}, z_{P n}\right)$ 。为论述方便, 仅在输 入与输出截面处分别施加一组外载荷。

以每个从动件为研究对象, 取分离体如图 $12 \mathrm{a}$ 所示, 从动件 $k_{i j}^{V}$ 受凸轮作用(接触)力 $\boldsymbol{F}_{i j}^{V}$ 、支承弹 簧回复力 $\boldsymbol{F}_{i j}^{V K}$ 和机架(移动导轨)的支反作用力 $\boldsymbol{F}_{i j}^{V f}$; 则由三力汇交平衡条件, 沿从动件移动方向平衡得 到 $\left|\boldsymbol{F}_{i j}^{V K}\right|=\left|\boldsymbol{F}_{i j}^{V}\right| \cos \alpha_{i j}^{V}$, 或

$$
\boldsymbol{F}_{i j}^{V}=\boldsymbol{n}_{i j}^{V}\left|\boldsymbol{F}_{i j}^{V K}\right| /\left(\left|\boldsymbol{n}_{i j}^{V}\right| \cos \alpha_{i j}^{V}\right)
$$

式中, $i, j=1,2 ; V=X, Y, Z, 10$ 个从动件对应 10 个方 程。其中 $\alpha_{i j}^{V}$ 为凸轮轮廓上接触点的压力角, 由式(15) 计算, $\boldsymbol{F}_{i j}^{V}$ 沿该接触点的凸轮曲面法向量 $\boldsymbol{n}_{i j}^{V}$ 方向。

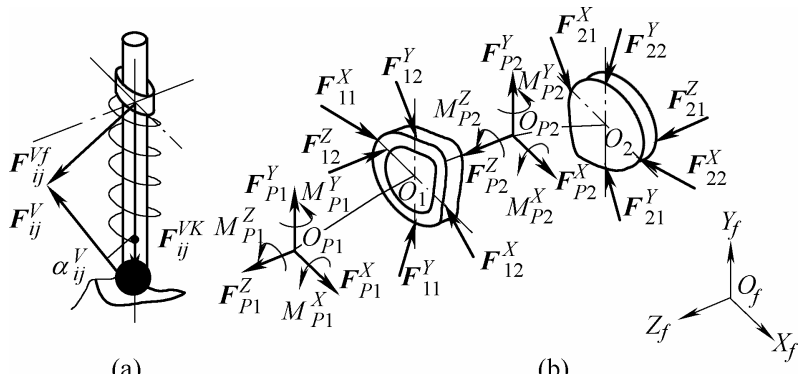

(a)

(b)

图 12 弹性几余机构中构件的受力分析

以凸轮及其旋转轴为研究对象, 取分离体如图 $12 \mathrm{~b}$ 所示, 受外载荷 $\boldsymbol{F}_{P 1} \sim \boldsymbol{F}_{P n} 、 \boldsymbol{M}_{P 1} \sim \boldsymbol{M}_{P n}$ 以及 10 个 从动件 $k_{i j}^{V}$ 在凸轮接触点的反作用力 $\boldsymbol{F}_{i j}^{V}$ 作用, 将机 架坐标系原点 $O_{f}$ 到力作用点(接触点) $P_{i j}^{V f}$ 的矢径记 为 $\boldsymbol{r}_{i j}^{V f}$, 如图 $12 \mathrm{~b}$ 所示, 有力、力矩矢量平衡方程

$$
\begin{gathered}
\sum_{i=1}^{2} \sum_{j=1}^{2}\left(\boldsymbol{F}_{i j}^{X}+\boldsymbol{F}_{i j}^{Y}+\boldsymbol{F}_{i j}^{Z}\right)+\sum_{k=1}^{n} \boldsymbol{F}_{P k}=\boldsymbol{0} \\
\sum_{i=1}^{2} \sum_{j=1}^{2}\left(\boldsymbol{F}_{i j}^{X} \boldsymbol{r}_{i j}^{X f}+\boldsymbol{F}_{i j}^{Y} \boldsymbol{r}_{i j}^{Y f}+\boldsymbol{F}_{i j}^{Z} \boldsymbol{r}_{i j}^{Z f}\right)+ \\
\sum_{k=1}^{n} \boldsymbol{F}_{P k} \boldsymbol{r}_{P k}+\sum_{k=1}^{n} \boldsymbol{M}_{P k}=\boldsymbol{0}
\end{gathered}
$$

上述力与力矩的矢量平衡方程展开共有 6 个标量平 衡方程, 外力和接触点矢量及压力角是已知量, 而 10 个从动件的作用力及其分量是未知量, 由式(19) 可以把 10 个从动件的作用力及其分量联系起来, 即 6 个方程 10 个待求未知量。 


\section{4 弹性冗余机构的物性方程}

在弹性冗余机构中用弹簧和弹性轴等效实际 回转副的弹性, 在此讨论在载荷作用下从动件弹簧 和弹性轴的变形与变形协调。依据刚度定义, 弹性 几余机构中每个从动件的载荷 $\boldsymbol{F}_{i j}^{V K}$ 与弹性位移 $\boldsymbol{\delta}_{i j}^{V}$ 有

$$
\boldsymbol{F}_{i j}^{V K}=k_{i j}^{V} \boldsymbol{\delta}_{i j}^{V} \quad i=1,2 \quad j=1,2 \quad V=X, Y, Z
$$

对具体回转副轴承结构而言, $k_{i j}^{V}$ 具有确定的函数关 系, 一般是非线性函数。在弹性几余机构中, 有 10 个从动件, 因而引入了 10 个方程, 与位移方程联立 成为变形协调方程, 但没有引入新的变量。

对于弹性冗余机构的旋转轴, 两端凸轮为几何 等效的刚性轮廓, 而回转轴本体属于弹性体, 两端 凸轮刚性轮廓之间还会产生弹性线位移和角位移, 如图 13 所示, 而这些弹性变形与旋转轴零件结构、 材料和载荷相关, 依据弹性理论 ${ }^{[18]}$, 用简化公式计 算, 对于两凸轮轮廓有

$$
\boldsymbol{\varepsilon}_{12}=\left(\varepsilon_{12}^{S X}, \varepsilon_{12}^{S Y}, \varepsilon_{12}^{S Z}, \varepsilon_{12}^{\theta X}, \varepsilon_{12}^{\theta Y}, \varepsilon_{12}^{\theta Z}\right)^{\mathrm{T}}=\sum_{n} \boldsymbol{C}_{S n} \boldsymbol{P}_{n}
$$

式中, $\varepsilon_{12}$ 为两端凸轮相对位移, $\boldsymbol{C}_{S n}$ 为凸轮连接轴 等效柔度, 由回转副旋转轴的弹性决定, 可用有限 元法计算, 并与轴上的等效载荷 $\boldsymbol{P}_{n}$ 一一对应, 其中 $\boldsymbol{P}_{n}=\left(\boldsymbol{F}_{P n}, \boldsymbol{M}_{P n}\right)^{\mathrm{T}}$ 。

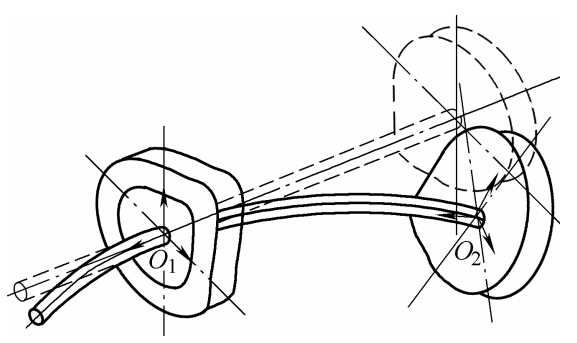

图 13 回转轴本体的弹性变形

式(23)展开共 6 个物性方程, 描述了两凸轮轮 廓及连接轴的相对弹性变形与载荷关系, 可求解两 端凸轮间的相对位置变化, 即两端凸轮之间的六个 增量参数 $\varepsilon_{12}^{S X} 、 \varepsilon_{12}^{S Y} 、 \varepsilon_{12}^{S Z} 、 \varepsilon_{12}^{\theta X} 、 \varepsilon_{12}^{\theta Y} 、 \varepsilon_{12}^{\theta Z}$, 其中 前三个为线位移, 后三个为角位移, 凸轮轮廓等效 几何曲面方程式(10)中 $\boldsymbol{R}_{2}=\boldsymbol{R}_{1}+\boldsymbol{\varepsilon}_{12}$ 。在实际回转副 中, 也可由物性方程式(23)反推出两端轴承及轴的 安装制造误差引起的附加载荷与变形, 从而由弹性 几余机构模型得出该误差对回转副精度特性的 影响。

\section{5 基本方程求解}

联立弹性冗余空间机构的位移方程式(16) (18)、静力平衡方程式(20)、(21)、物性方程式(22)、
(23), 称为弹性冗余机构的基本方程, 展开共 32 个 方程和 32 个独立待求变量, 可以求解出等效弹性几 余空间机构的从动件位移参数与力参数; 在实际回 转副中, 当以回转轴转角作为输入时, $\theta_{z 1}$ 为自变量, 而力矩平衡方程 $\Sigma M_{z}=0$ 变成外力矩与摩擦力矩的 平衡, 与上述独立变量无关, 即有效方程数与独立 待求变量数均减少为 31 个。

上述位移方程中的 $\boldsymbol{r}_{i j}^{V}$ 与凸轮轮廓形状及凸轮 在机架坐标系下的位置相关，一般无显示表达式， 而变形协调方程中的 $k_{i j}^{V}$ 为非线性, 上述基本方程需 要通过数值方法联立求解, 求解步骤如下。

（1）假定等效凸轮中心初始位置 $\boldsymbol{R}_{i}=\boldsymbol{R}_{i 0}$, 由凸 轮轮廓方程式(10)与从动件移动导轨轴线方程式 $(11) \sim(13)$, 求解凸轮与从动件的接触点 $p_{i j}^{V \mathrm{f}}$ 坐标。

(2) 由接触点 $p_{i j}^{V \mathrm{ff}}$ 坐标与凸轮中心位置 $\boldsymbol{R}_{i}$ 计算 矢量 $\boldsymbol{r}_{i j}^{V} 、 \boldsymbol{r}_{i}$, 再由位移方程式 $(16) \sim(18)$ 求解从动 件位移 $\boldsymbol{\delta}_{i j}^{V}$ 。

(3) 由物性方程(22)求从动件载荷 $\boldsymbol{F}_{i j}^{V K}$, 由方程 式(14)、(15)求解接触点法矢量与接触角, 代入式(19) 计算凸轮载荷 $\boldsymbol{F}_{i j}^{V}$ 。

（4）由物性方程式(23)计算两端凸轮位移增量 $\boldsymbol{\varepsilon}_{12}$, 由平衡方程式(20)、(21)左端计算当前位置下 的载荷不平衡量, 得到由位移增量及载荷不平衡引 起的凸轮位置变化量, 确定新的凸轮位置 $\boldsymbol{R}_{i}$, 并返 回第(1)步, 当载荷不平衡量满足精度要求时停止迭 代, 最后一次计算结果即为上述方程的求解结果。

\section{4 回转副滚动轴承结构精度特性示例}

\section{1 回转副滚动轴承结构与参数}

以图 14 所示的两端采用深沟球轴承支承的典 型回转副结构为例, 两端轴承型号均为 P0 级 6312 轴承, 回转副及轴承的基本参数如表 1 所示, 截面 $O_{P 1}$ 为输出截面(位移测试截面), 截面 $O_{P 2}$ 为输入截 面(加载截面), $L_{1} 、 L_{2}$ 分别为截面 $O_{P 1}$ 、截面 $O_{P 2}$ 与 截面 $O_{1}$ 的距离, $L_{3}$ 为轴承支承跨距。本例以数据 说明弹性冗余空间机构的建模过程及其对回转副精 度特性的影响, 详细参数确定与求解另文论述。

图 14 中回转副的等效弹性几余空间机构模型 如图 9a 所示, 根据回转副及轴承的结构参数, 对应 机构结构参数有 $L_{D i}=117.225 \mathrm{~mm} 、 L_{B i}=15.50 \mathrm{~mm}$ 、 $L_{S}=210 \mathrm{~mm} 、\left|\boldsymbol{L}_{i j}^{X}\right|=\left|\boldsymbol{L}_{i j}^{Y}\right|=22.225 \mathrm{~mm} 、\left|\boldsymbol{L}_{i j}^{Z}\right|=15.50$ $\mathrm{mm}$ 。 


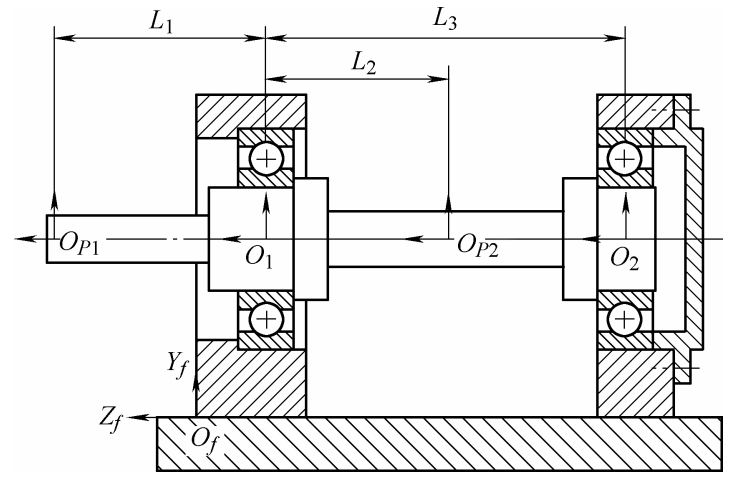

图 14 回转副轴承结构简图

表 1 回转副轴承基本结构参数表

\begin{tabular}{lc||lc}
\hline \multicolumn{1}{c||}{ 参数 } & 数值 & \multicolumn{1}{|c}{ 参数 } & 数值 \\
\hline 轴承内径 $d / \mathrm{mm}$ & 60.0 & 外圈沟曲率系数 $f_{e}$ & 0.525 \\
轴承外径 $D / \mathrm{mm}$ & 130.0 & 初始径向游隙 $u_{r} / \mathrm{mm}$ & 0.018 \\
内圈宽度 $B / \mathrm{mm}$ & 31.0 & 滚珠数 $Z$ & 8 \\
外圈宽度 $C / \mathrm{mm}$ & 31.0 & $O_{P 1}$ 与 $O_{1}$ 距离 $L_{1} / \mathrm{mm}$ & 125.0 \\
滚珠直径 $D_{w} / \mathrm{mm}$ & 22.225 & $O_{P 2}$ 与 $O_{1}$ 距离 $L_{2} / \mathrm{mm}$ & 105.0 \\
内圈沟曲率系数 $f_{i}$ & 0.515 & 轴承跨距 $L_{3} / \mathrm{mm}$ & 210.0 \\
\hline
\end{tabular}

\section{2 回转副轴承支承结构的约束刚度}

如第 1.2 节所述, 轴承支承刚度是轴承座、轴 承、轴等零部件及其配合面刚度的综合, 此处分为 两部分近似计算, 轴、轴承座的结构刚度通过有限 元法计算, 分析不同载荷下各个方向上零件的弹性 变形来计算其等效刚度参数, 结果如表 2 所示。

轴承刚度可采用文献[15]中的公式近似计算, 轴承结构参数取表 1 中数据。设 $\alpha$ 为轴承的初始接 触角, 本文中近似计算忽略由于滚珠及内外滚道的
弹性变形而引起的实际接触角变化, 仅考虑轴承的 几何参数，初始接触角计算如下 ${ }^{[15]}$

表 2 轴承座与轴的结构刚度 $10^{5} \mathrm{~N} / \mathrm{mm}$

\begin{tabular}{ccccccc}
\hline 刚度 & $k_{S 1}^{X}$ & $k_{S 2}^{X}$ & $k_{S 1}^{Y}$ & $k_{S 2}^{Y}$ & $k_{S 1}^{Z}$ & $k_{S 2}^{Z}$ \\
\hline 左端 & 8.50 & 8.50 & 6.06 & 10.10 & 无约束 & 2.33 \\
右端 & 5.64 & 5.64 & 4.45 & 7.27 & 1.19 & 无约束 \\
\hline
\end{tabular}

$$
\cos \alpha=1-\frac{u_{r}}{2 D_{w}\left(f_{e}+f_{i}-1\right)}=0.9899
$$

将接触角代入刚度公式 ${ }^{[15]}$, 得到轴承刚度与位移的 关系: $k_{B}^{r}=1190282 \delta_{r}^{1 / 2} 、 k_{B}^{a}=46568 \delta_{a}^{1 / 2}$, 即 $V=X$, $Y$ 时, $k_{B j}^{V}=k_{B}^{r}, V=Z$ 时, $k_{B j}^{V}=k_{B}^{a}$; 两端轴承刚度相 同。在此以弹簧串联刚度公式计算两部分的综合刚 度

$$
\frac{1}{k_{j}^{V}}=\frac{1}{k_{B j}^{V}}+\frac{1}{k_{S j}^{V}}
$$

分别将表 2 中计算的结构刚度与上述轴承刚度 公式代入式(24), 得到各支承弹簧的刚度计算式。

\section{3 回转副轴承结构的几何特征等效}

依据第 2.1 节中几何特征等效方法, 对图 14 所 示回转副的两端轴承及其支承结构的几何误差进行 等效。以下计算以左端轴承为例, 首先分截面测量 轴承支承结构中各相关零件表面轮廓，再以式(3)中 傅里叶级数拟合各截面形状曲线, 其中各零件表面 中心截面拟合的相关参数如表 3 所示，拟合置信区 间为 $95 \%$ ，其他截面数据本文中不再列出。

\begin{tabular}{|c|c|c|c|c|c|c|c|c|c|c|c|c|c|c|c|}
\hline & $r_{0} / \mathrm{mm}$ & $a_{0} / \mu \mathrm{m}$ & $a_{1} / \mu \mathrm{m}$ & $a_{2} / \mu \mathrm{m}$ & $a_{3} / \mu \mathrm{m}$ & $a_{4} / \mu \mathrm{m}$ & $a_{5} / \mu \mathrm{m}$ & $a_{6} / \mu \mathrm{m}$ & $b_{1} / \mu \mathrm{m}$ & $b_{2} / \mu \mathrm{m}$ & $b_{3} / \mu \mathrm{m}$ & $b_{4} / \mu \mathrm{m}$ & $b_{5} / \mu \mathrm{m}$ & $b_{6} / \mu \mathrm{m}$ & $w / \mathrm{rad}$ \\
\hline 轴表面 & 30.0000 & 6.00 & -0.58 & 0.19 & 0.01 & 0.08 & -0.03 & 0 & -2.07 & -0.12 & 0.22 & -0.09 & 0.13 & 0 & 1.01 \\
\hline $\begin{array}{l}\text { 内圈内 } \\
\text { 孔面 }\end{array}$ & 30.0000 & -0.89 & -0.46 & -0.45 & -0.16 & -0.15 & -0.07 & -0.09 & 0.16 & 0.69 & -0.23 & 0.06 & 0.05 & 0.09 & 1.02 \\
\hline $\begin{array}{l}\text { 外圈外 } \\
\text { 圆面 }\end{array}$ & 65.0000 & 2.08 & 0.05 & -0.17 & -0.35 & -0.01 & 0.35 & 0 & -0.04 & 0.07 & 0.24 & 0 & -0.12 & 0.01 & 0.99 \\
\hline $\begin{array}{l}\text { 内圈 } \\
\text { 滚道 }\end{array}$ & 36.3825 & 0.01 & 1.96 & -0.05 & -0.07 & -0.02 & -0.04 & -0.02 & -4.13 & 0.39 & -0.50 & 0.22 & -0.01 & -0.03 & 1.02 \\
\hline $\begin{array}{l}\text { 外圈 } \\
\text { 滚道 }\end{array}$ & 58.6165 & 0.10 & 0.03 & 0.25 & -1.05 & -0.61 & 0.24 & 0.26 & 1.12 & 0.52 & -0.06 & -0.08 & 0.54 & 0.30 & 1.03 \\
\hline
\end{tabular}

表 3 轴承支承各表面中间截面形状拟合傅里叶级数系数

算例中包含两个静态联接面, 其中轴承外圈与 轴承座之间为间隙配合, 不改变轴承外圈滚道形状, 即无弹性映射; 而轴承内圈与轴之间的过盈配合, 考虑实际轴与内圈之间的过盈量, 以内孔面与轴表 面几何特征的等效曲面建立有限元实体模型, 计算 轴与内圈联接面几何特征的弹性映射关系(含过盈
量影响), 限于篇幅, 详细计算过程作者将另文论述。 在回转副零件表面几何特征分析等效以及静 配合弹性传递与映射后, 可得到外圈滚道等效曲面、 内圈滚道等效曲面和滚动体球面, 共 8 个球面、2 个滚道等效曲面。再按运动联接面的几何特征映射 进行编程数值计算, 得到满足几何约束与载荷稳定 
条件下的等效凸轮轮廓。在此绘出从动件 $k_{11}^{X}$ 与 $k_{11}^{Y}$ 所对应凸轮的中心截面形状, 分别如图 $15 \mathrm{a} 、 15 \mathrm{~b}$ 所示, 图 15 中凸轮轮廓波动放大 2000 倍显示, 凸 轮轮廓存在跳动是由于实际轴承中滚珠分布不连 续, 其他 8 个从动件对应的凸轮数据不再一一列出。

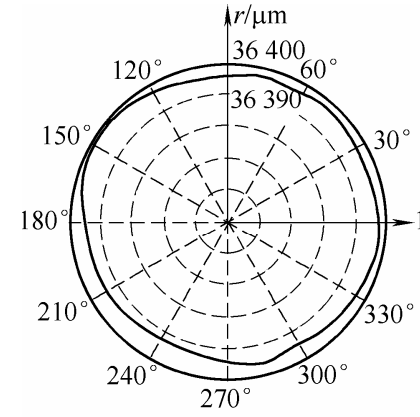

(a) $k_{11}^{X}$

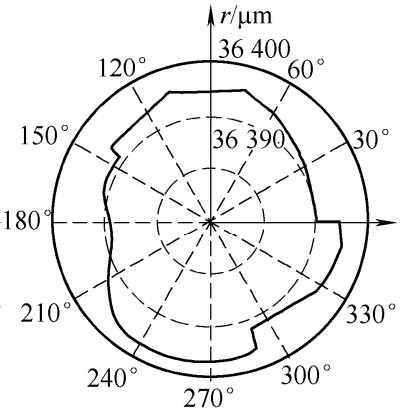

(b) $k_{11}^{Y}$
图 $15 k_{11}^{X}$ 与 $k_{11}^{Y}$ 对应等效凸轮中心截面几何轮廓曲线

\section{4 回转副精度特性计算示例}

\subsection{1 弹性冗余机构基本方程的特定位置解}

旋转轴转角固定 $\left(\theta_{z 1}=0\right)$, 输入截面 $O_{P 2}$ 施加载 荷 $\boldsymbol{F}_{P 2}=(1100 \mathrm{~N}, 0,0)$, 计算得两端凸轮在机架坐标 系下的位置参数 $\boldsymbol{R}_{i}=\left(x_{i}, y_{i}, z_{i}, \theta_{x i}, \theta_{y i}, \theta_{z i}\right)$, 其中 $\boldsymbol{R}_{1}=$ $\left(10.6,7.7,-41000,7.5 \times 10^{-5},-2.3 \times 10^{-4}, 0\right) 、 \boldsymbol{R}_{2}=(14.4$, $\left.-4.4,-251000,7.5 \times 10^{-5}, 1.9 \times 10^{-4}, 0\right)$, 在此约定 $\boldsymbol{R}_{i}$ 中位移量单位取 $\mu \mathrm{m}$, 角度量单位取 $\mathrm{rad}$; 各从动件 的静力与位移结果如表 4 所示。

表 4 从动件静力与位移求解结果

\begin{tabular}{lcccccccccc}
\hline 从动件 & $k_{11}^{X}$ & $k_{12}^{X}$ & $k_{11}^{Y}$ & $k_{12}^{Y}$ & $k_{12}^{Z}$ & $k_{21}^{X}$ & $k_{22}^{X}$ & $k_{21}^{Y}$ & $k_{22}^{Y}$ & $k_{21}^{Z}$ \\
\hline 载荷 $/ \mathrm{N}$ & 0 & 550 & -35 & 0 & 1 & 0 & 550 & -21 & 0 & -1 \\
& & & & & & & & & & \\
位移 $/ \mu \mathrm{m}$ & 0 & 6.3 & -1.5 & 0 & 0.6 & 0 & 6.3 & -1.1 & 0 & -0.6 \\
\hline
\end{tabular}

4.4.2 定常载荷下回转副任意转角的精度特性计算 旋转轴转角 $\theta_{z 1}$ 从 $0^{\circ}$ 变化到 $360^{\circ}$, 仅施加轴承 轴向预紧载荷 $500 \mathrm{~N}$, 回转轴输出截面 $O_{P 1}$ 的形心 轨迹与法线偏角如图 16a、16b 所示，其形心在 $X$ 方向位移分量随旋转轴转角变化曲线如图 17 所示。

图 17 中形心轨迹不连续是由于回转副滚动轴 承中滚珠分布不连续, 即经轴承联接面映射得到内 圈运动轨迹存在跳动, 进而导致等效凸轮轮廓不 连续。

\subsection{3 变载荷下回转副特定转角精度特性计算}

旋转轴转角固定 $\left(\theta_{z 1}=320^{\circ}\right)$, 输入截面 $O_{P 2}$ 施加 沿 $X$ 方向梯度变化载荷 $(0 \sim 3000 \mathrm{~N})$, 以弹性几余空 间机构模型分别计算在单一参数(其他参数均为理 想值)及各参数综合作用下的回转副精度变化曲线, 如图 18 所示, 其中: (1) 当仅考虑零件几何误差因

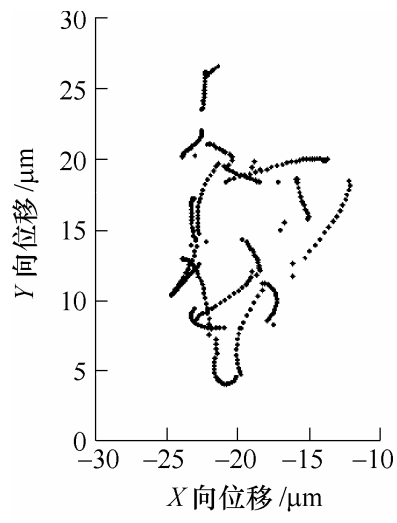

(a) 输出截面形心轨迹

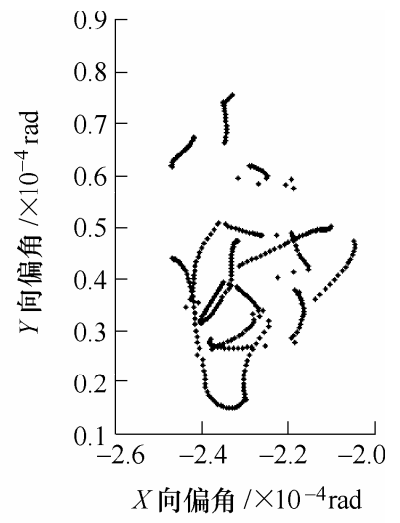

(b) 输出截面法线偏角
图 16 回转副输出截面形心轨迹与法线偏角变化曲线

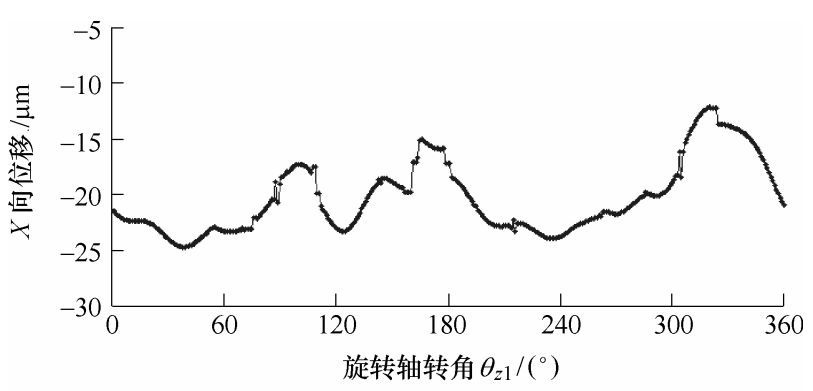

图 17 定常载荷下回转副位置 $X$ 方向变化曲线

素并以等效凸轮轮廓形状体现时, 令轴刚度和从动 件弹簧刚度为无穷大, 计算结果为曲线 $a_{1}$; (2) 当 仅考虑轴承刚度因素并由从动件弹簧刚度表示时, 轴刚度取无穷大，而等效凸轮形状分别为理想圆 $r_{0}$ 和理想平面 $z_{0}$, 结果为曲线 $a_{2}$; (3) 当仅考虑轴刚 度因素时，从动件弹簧刚度取无穷大，等效凸轮形 状为理想圆 $r_{0}$ 和理想平面 $z_{0}$, 计算结果为曲线 $a_{3}$; (4) 几何误差、轴承刚度、轴刚度三种因素均取实际 参数, 综合结果为曲线 $a_{4}$ 。

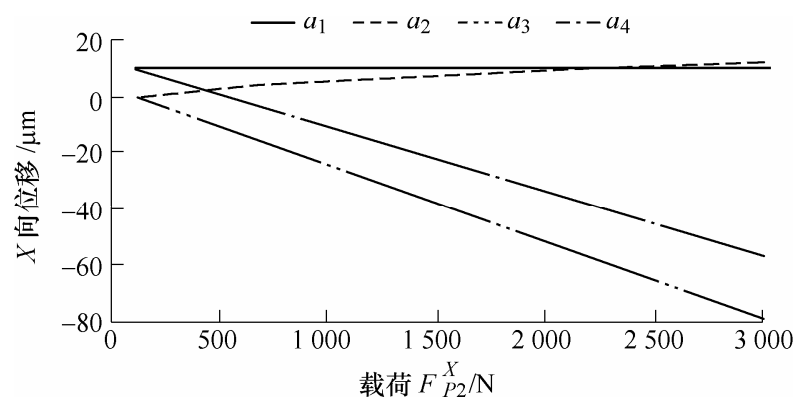

图 18 变载荷回转副特定转角精度特性曲线

\subsection{4 变载荷下回转副任意转角精度特性计算}

旋转轴转角 $\theta_{z 1}$ 从 0 变化到 $360^{\circ}$, 输入截面 $O_{P 2}$ 施加沿 $X$ 方向的正弦变化载荷 $1100+400 \sin \theta(\mathrm{N})$, 同样以弹性冗余空间机构模型求解在单一参数(其 他参数均为理想值)及各参数综合作用下的回转副 精度特性, 计算结果如图 19 所示, 其中曲线 $a_{1}$ 、 $a_{2} 、 a_{3} 、 a_{4}$ 计算条件同上。 


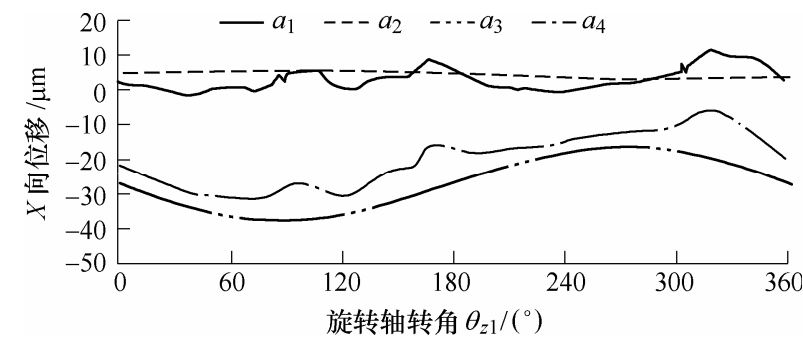

图 19 变载荷下回转副任意位置的精度变化特性曲线

由上述计算结果可见载荷、位置不同时, 各个 参数对回转副精度的影响程度并不相同。本算例中, 在载荷较小时, 回转副精度主要受轴承等零件的几 何精度影响; 载荷较大时, 轴承支承结构的弹性变 形以及轴的变形占主要部分, 且轴的变形比例增大, 本节算例与下节试验设计有意将旋转轴刚度设计偏 小，显示出回转副精度的薄弱环节。当采用不同的 回转副结构与刚度参数时其影响程度也不相同, 可 利用该模型计算, 为回转副的精度特性分析与精度 设计提供依据。

\section{5 回转副精度模型的试验测试}

本文设计了回转副轴承结构精度模型的简易 试验测试装置, 如图 20 所示, 径向施加载荷, 轴向 施加预紧, 并采用力传感器(直线度 \pm 0.02 , 量程 $20 \mathrm{kN}$ ) 测量记录, 回转轴角位移采用光棚编码器(每转脉冲 数 1024 , 相对角度误差不大于 $0.07^{\circ}$ ), 弹性变形及 线位移采用光栅位移传感器(示值误差不大于 0.3 $\mu \mathrm{m})$ 测量。试验记录旋转轴上输出截面径向跳动, 采用三点法 ${ }^{[19]}$ 测试并处理数据, 去除测试轴表面形 状误差对结果的影响。对应上述的计算分析内容, 分别进行试验测试, 计算数据与试验数据结果如图 21 24 所示, $b_{1}$ 为弹性尣余空间机构的计算结果, $b_{2}$ 为试验测试曲线, $b_{3}$ 为 $b_{2}$ 的拟合曲线。图 21 为 旋转轴在 $\theta_{z 1}=320^{\circ}$ 时, 径向载荷变化 $(0 \sim 3000 \mathrm{~N})$ 时输出截面 $O_{P 1}$ 形心点 $X$ 方向位移。

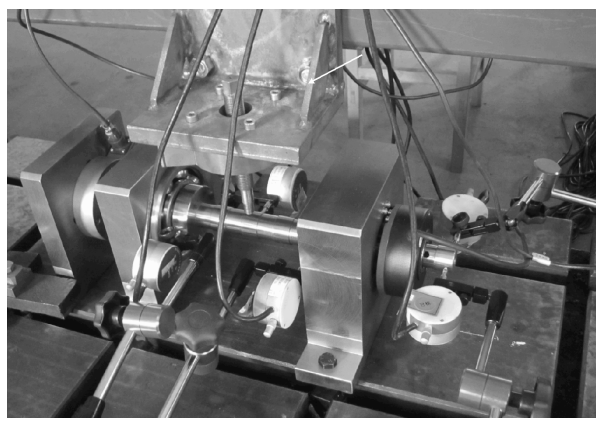

图 20 回转副精度模型的试验测试装置

在轴向预紧力 $500 \mathrm{~N}$ 下, 无径向载荷时输出截 面 $O_{P 1}$ 形心点 $X$ 方向位移如图 22 所示。

径向载荷恒定为 $\boldsymbol{F}_{P 2}=(1100,0,0) \mathrm{N}$ 时输出截面 $O_{P 1}$ 形心点 $X$ 向位移如图 23 所示。

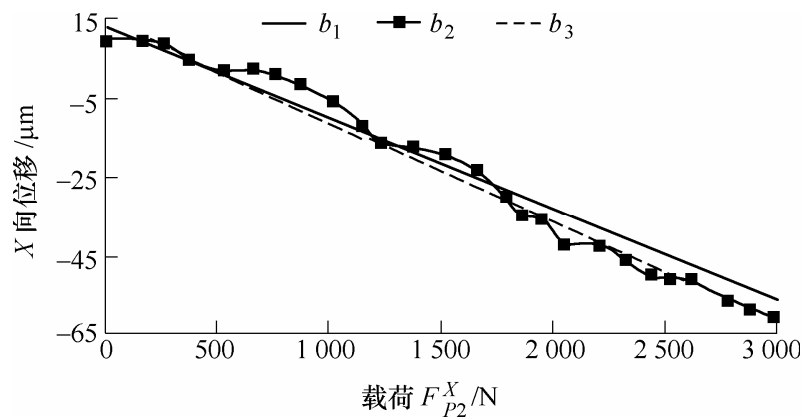

图 21 变载荷下固定位置时回转副精度计算与试验结果

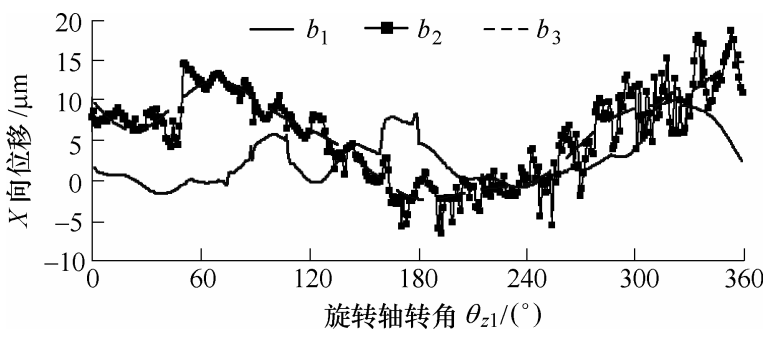

图 22 预紧力 $500 \mathrm{~N}$ 时回转副任意角度精度计算与试验结果

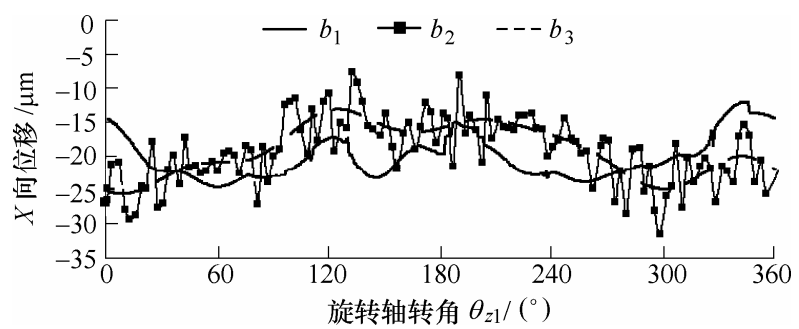

图 23 定常载荷下回转副任意角度的精度计算与试验结果

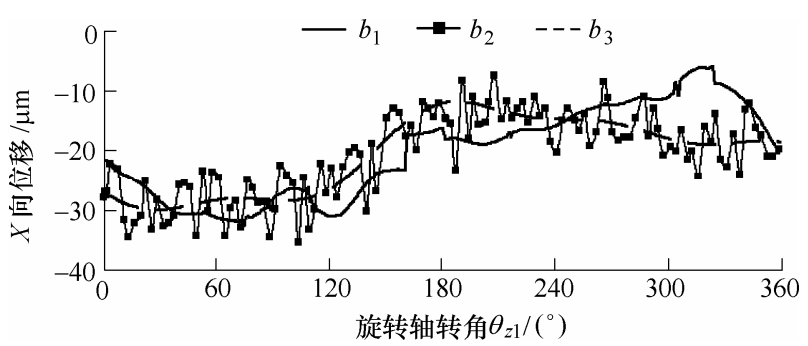

图 24 变载荷下回转副任意角度精度计算与试验结果

径向载荷按正弦规律变化 $1100+400 \sin \theta(\mathrm{N})$ 时输出截面 $O_{P 1}$ 形心点 $X$ 向位移如图 24 所示。

上述计算与试验数据初步表明, 实际回转副的 精度受载荷、几何、物理等因素的耦合作用, 试验 与计算结果的位移变化趋势相近，波动范围基本一 致, 说明利用本文中的机构模型可较真实反映实际 回转副精度以及变化特性, 而试验数据曲线与计算 曲线形状差别是由于相位计算情况与试验装置中两 端轴承内外圈之间实际相位不吻合所致, 同时试验 条件未能较好避免振动等环境干扰因素影响。

\section{5 结论}

（1）提出了弹性冗余空间机构模型，建立了回 
转副精度与其轴承零件结构、几何特征、材料与结 构弹性的内在联系, 弹性元余空间机构的位移方程、 静力平衡方程和物性方程体现几何误差特征与弹性 变形的相互转化与耦合作用, 较好地表述回转副的 精度及其特性。

(2) 回转副精度模型所对应的弹性几余空间机 构是一种新型机构, 具有弹性和午余运动自由度。

(3) 回转副轴承结构的材料弹性约束与预紧可 用直动从动件弹簧刚度及初始位置描述; 回转副轴 承结构及零件间的安装与配合误差可用凸轮从动件 支承弹簧在机架上位置反映; 回转副轴承零件结构 的几何误差特征以等效刚性凸轮轮廓曲面体现。

\section{参 考 文 献}

[1] KOTHALKAR S A, YAJNIK K S. The effect of play in the joints of a function generating mechanism[J]. Journal of Mechanisms, 1970，5(4): 521-532.

[2] 徐卫良, 张启先. 空间机构运动误差的概率分析和蒙特 卡罗模拟[J]. 东南大学学报, 1988, 18(6): 1-8.

XU Weiliang, ZHANG Qixian. Probability analysis and Monte Carlo simulation of the kinematic errors in a spatial linkage[J]. Journal of Southeast University, 1988, 18(6): $1-8$.

[3] SERKOW N A. Primary deviations of mechanism links with sliding and revolute pairs[J]. Journal of Machinery Manufacture and Reliablity, 2011, 40(2): 107-112.

[4] LEE S J, GILMORE B J. The determination of the probabilistic properties of velocities and accelerations in kinematic chains with uncertainty[J]. ASME Journal of Mechanical Design, 1991, 113(1): 84-90.

[5] JOSCOWICZ L, SACKS E, SRINIVASAN V. Kinematic tolerance analysis[J]. Computer-Aided Design, 1997, 29(2): 147-157.

[6] ZHANG Guojun, CHENG Qiang, SHAO Xinyu, et al. Accuracy analysis for planar linkage with multiple clear-ances at turning pairs[J]. Chinese Journal of Mechanical Engneering, 2008，21(2): 36-41.

[7] 许立新, 李永刚, 李充宁, 等. 轴承间隙及柔性特征对 机构动态误差的影响分析 [J]. 机械工程学报, 2012, 48(7): 30-36.

XU Lixin, LI Yonggang, LI Chongning, et al. Effects of bearing clearance and flexibility on the dynamic errors of mechanisms[J]. Journal of Mechanical Engineering, 2012, 48(7): 30-36.

[8] 吴玉光, 张根源, 李春光. 夹具定位误差分析自动建模 方法[J]. 机械工程学报, 2012, 48(5): 171-179.
WU Yuguang, ZHANG Genyuan, LI Chunguang. Approach to automated modeling of fixture location errors analysis[J]. Journal of Mechanical Engineering, 2012, 48(5): 171-179.

[9] 李哲. 含间隙弹性平面连杆机构动力分析 [J]. 机械工 程学报, 1994, 30(增): 134-139.

LI Zhe. Dymamic analysis of elastic planar linkages with clearances[J]. Chinese Journal of Mechanical Engineering, 1994, 30(Suppl.): 134-139.

[10] 焦国太, 余跃庆, 梁浩. 机器人位姿误差的结构矩阵分 析方法[J]. 应用基础与工程科学学报, 2001, 9(2-3): 259-265.

JIAO Guotai, YU Yueqing, LIANG Hao. The application of matrix-structural method to the error analysis of robots[J]. Journal of Basic Science and Engineering, 2001, 9(2-3): 259-265.

[11] KIRIDENA V, FERREIRA P M. Mapping the effects of positioning errors on the volumetric accuracy of five-axis $\mathrm{CNC}$ machine tools[J]. International Journal of Machine Tools \& Manufacture, 1993, 33(3): 417-437.

[12] 杨建国. 加工中心的几何误差和热误差综合补偿模型 [J]. 计量学报, 2001, 22(2): 90-94.

YANG Jianguo. Geometric and thermal error synthesis model for machining centers[J]. Acta Metrologica Sinica, 2001, 22(2): 90-94.

[13] SUN Tao, SONG Yimin, LI Yonggang, et al. Separation of comprehensive geometrical errors of a 3-DOF parallel manipulator based on Jacobian matrix and its sensitivity analysis with Monte-Carlo method[J]. Chinese Journal of Mechanical Engineering, 2011, 24(3): 406-413.

[14] 刘显军, 洪军, 朱永生, 等. 多支承轴系轴承受力与刚 度的有限元迭代计算方法 [J]. 西安交通大学学报, 2010, 44(11): 41-45.

LIU Xianjun, HONG Jun, ZHU Yongsheng, et al. Iterative method to solve beating's force and stiffness for a multi-support spindle system based on finite analysis[J]. Journal of Xi' an Jiaotong University, 2010, 44(11): 41-45.

[15] 万长森. 滚动轴承的分析方法[M]. 北京: 机械工业出 版社, 1987.

WAN Changsen. Analysis of rolling bearings[M]. Beijing: China Machine Press, 1987.

[16] 王硕桂, 夏源明. 过盈配合量和预紧力对高速角接触球 轴承刚度的影响[J]. 中国科学技术大学学报, 2006, 36(12): 1315-1320.

WANG Shuogui, XIA Yuanming. Effect of the 
interference fit and axial preload in the stiffness of the high-speed angular contact ball bearing[J]. Journal of University of Science and Technology of China, 2006, 36(12): 1315-1320.

[17] HUNT K H. Kinematic geometry of mechanisms[M]. Oxford: Oxford University Press, 1978.

[18] 陈忠安, 王静. 材料力学 $[\mathrm{M}]$. 北京: 北京大学出版社, 2009.

CHEN Zhongan, WANG Jing. Strength of material[M].
Beijing: Peking University Press, 2009.

[19] 魏源迁, 庞学慧, 白恩远. 三点法误差分离技术理论分 析[J]. 计量学报, 1991，12(3): 199-205.

WEI Yuanqian, PANG Xuehui, BAI Enyuan. Theoretical analyses of error separation techniques[J]. Acta Metrologica Sinica, 1991, 12(3): 199-205.

作者简介: 王德伦, 男, 1958 年出生, 博士, 教授, 博士研究生导师。 主要从事机构学及机械装备数字化设计方法研究。

E-mail: dlunwang@dlut.edu.cn 